\title{
Konsensusempfehlungen deutscher Experten Management der Therapie mit Idelalisib
}

Idelalisib, die erste Substanz in der Wirkklasse der Phosphatidylinositol 3-Kinase delta (PI3Kס)-Inhibitoren, ist in Deutschland für die Therapie von Patienten mit chronischer lymphatischer Leukämie (CLL) und follikulärem Lymphom (FL) zugelassen. Im Rahmen eines Expertenmeetings wurden Empfehlungen für das Therapiemanagement erarbeitet, um das Nutzen-Risiko-Profil von Idelalisib weiter zu diskutieren. In klinischen Studien [2] hat Idelalisib in den zugelassenen Indikationen eine hohe Wirksamkeit gezeigt. Doch wie jede effektive Behandlung kann auch die Idelalisib-Therapie mit Nebenwirkungen assoziiert sein, deren Management im klinischen Alltag beherrscht werden muss. Als wichtige potenzielle Nebenwirkungen wurden in diesem Zusammenhang insbesondere Kolitiden, Infektionen unter anderem mit dem Cytomegalievirus (CMV) und Pneumocystis jirovecii-Pneumonien (PJP) sowie nichtinfektiöse Pneumonitiden definiert.

Mit Idelalisib $\left(\right.$ Zydelig $^{\circledR}$ ) steht seit rund 2 Jahren der erste selektive PI3K $\delta$-Inhibitor für die Therapie von Patienten mit CLL und FL zur Verfügung. Zur Behandlung von erwachsenen Patienten mit CLL wird Zydelig ${ }^{\circledR}$ in Kombination mit einem monoklonalen Anti-CD20-Antikörper (Rituximab oder Ofatumumab) angewendet: - wenn diese mindestens eine vorangehende Therapie erhalten haben,

- als Erstlinientherapie bei Vorliegen einer 17p-Deletion oder einer TP53-Mutation bei Patienten, für die keine anderen Therapien geeignet sind [1].

Als Monotherapie wird Zydelig ${ }^{\circledR}$ zur Behandlung von erwachsenen Patienten mit FL, das nach 2 vorausgegangenen Therapielinien refraktär ist, angewendet [1]. PI3K $\delta$ ist Bestandteil mehrerer, von Oberflächenrezeptoren wie dem B-Zell-Rezeptor ausgehender intrazellulärer Signalwege. Ihre Aktivierung führt zur Phosphorylierung von AKT, einem zentralen Knotenpunkt verschiedener Signalkaskaden, die an der Regulation von Zellproliferation, Motilität und Überleben beteiligt sind. Da die PI3K $\delta$ bei B-Zell-Malignomen überaktiviert ist, bietet sie sich als therapeutisches Target für die Behandlung dieser Erkrankungen an.

\section{Effektiv bei CLL und FL}

Die Erstzulassung von Idelalisib in der CLL beruht auf einer placebokontrollierten Phase-III-Studie (GS-US-312-0116) an 220 Patienten mit progredienter Erkrankung [1]. Verglichen wurde die Therapie mit Rituximab (plus Placebo) im Kontrollarm mit der Kombination Rituximab plus Idelalisib im Verumarm. Bereits mit der ersten Interimsanalyse ergab sich für den Arm mit Idelalisib eine signifikante Verbesserung des progressionsfreien Überlebens (PFS) mit einer Reduktion des Progressionsrisikos um relativ 85\% (Hazard Ratio (HR) 0,15; $\mathrm{p}<0,0001)$ [2]. Der Median im PFS war bei den mit Idelalisib behandelten Patienten zu diesem Zeitpunkt noch nicht erreicht; im Placeboarm lag er bei 5,5 Monaten [2]. Laut dem Ende 2014 vorgestellten Update der Studie betrug das PFS im Idelalisib-Arm 19,4 Monate gegenüber 7,3 Monaten im Kontrollarm [3]. In der aktualisierten Analyse war der Median im Gesamtüberleben unter Idelalisib noch immer nicht erreicht. Bei den Kontrollpatienten lag der Median bei 20,8 Monaten (HR 0,34; $\mathrm{p}=0,0001$ ). Häufigste Nebenwirkungen (Grad 3-4) waren laut Studien-Update Neutropenien (41\%), Diarrhoe und Kolitiden (16\%), Thrombozytopenien (14\%) sowie Pneumonien (13\%) [3]. Die Ergebnisse führten Mitte September 2014 zur EU-Zulassung von Idelalisib. Seit Oktober 2016 ist Idelalisib auch in der Kombination mit dem Anti-CD20-Antikörper Ofatumumab für die Behandlung von CLL-Patienten zugelassen [1]. Basis der Zulas- sung von Idelalisib beim FL war eine offene 1-armige Phase-II-Studie bei 72 doppelt refraktären Patienten (refraktär gegenüber Rituximab und einem Alkylanz) [4]. Die durch ein unabhängiges Review-Komitee ermittelte Ansprechrate (primärer Endpunkt) betrug 55,6\%; darunter gab es komplette Remissionen (14\%) [4]. Responder sprachen median 12,5 Monate auf Idelalisib an. Das PFS liegt bei median 11 Monaten, das Gesamtüberleben bei 88,1\% nach 12 Monaten und $69,8 \%$ nach 24 Monaten [4]. Eine schwere Neutropenie vom Grad $\geq 3$ trat bei 22,2\% der Studienteilnehmer auf. Diarrhoe (Grad $\geq 3$ ) wurde bei 13,9\% der Patienten beobachtet [4].

\section{EMA bestätigte Idelalisilb ein positives Nutzen-Risiko-Verhältnis}

Im März 2016 wurde von der Europäischen Arzneimittel-Agentur (EMA) ein Risikobewertungsverfahren für Idelalisib (Bewertungsverfahren nach Artikel 20 der Verordnung (EG) Nr. 726/2004) eingeleitet und im September abgeschlossen [5]. Hintergrund dieses Verfahrens waren Signale aus 3 laufenden Phase-III-Studien, nach denen im Behandlungsarm unter Idelalisib eine erhöhte Anzahl infektionsbedingter Todesfälle im Vergleich zum Kontrollarm auftraten. Die zusätzlichen Todesfälle waren hauptsächlich auf Infektionen, einschließlich Infektionen mit PJP und CMV, zurückzuführen [6]. Diese inzwischen abgebrochenen Studien prüften Idelalisib außerhalb der derzeit zugelassenen Indikationen als Zusatztherapie zur standardmäßigen CLL-Erstlinientherapie bzw. in frühen Therapielinien beim indolenten Non-Hodgkin-Lymphom (iNHL) und beim kleinzelligen lymphozytischen Lymphom (SLL). Nach Abschluss des Risikobewertungsverfahrens ist Idelalisib zugelassen in Kombination mit einem monoklonalen Anti-CD20-Antikörper zur Behandlung erwachsener CLL-Patienten, die mindestens eine vorangehende Therapie erhalten haben, oder als Erstlinientherapie bei Vorliegen einer 17p-Deletion oder einer TP53-Mutation bei Patienten, für die keine anderen Therapien geeignet sind [1]. Daneben ist Idelalisib indiziert als Monotherapie zur Behandlung von erwachsenen Patienten mit FL, das nach 2 vorausgegangenen Therapielinien refraktär ist [1].

\section{Internationale Empfehlungen zum Nebenwirkungs- management durch Expertenpanel erarbeitet}

Bereits 2015 wurden Empfehlungen eines amerikanischen Expertenpanels zum Nebenwirkungsmanagement der Idelalisib-Therapie publiziert [7]. Auf einem deutschen Expertenmeeting (Tab. 1) wurden im April 2016 die zu Idelalisib vorliegenden Erfahrungen bei CLL und FL kritisch bewertet und Empfehlungen zum Therapie-Management erarbeitet.
S. Karger

Medical and Scientific Publishers

Basel · Freiburg · Paris · London

New York · Chennai · New Delhi

Bangkok · Beijing · Shanghai -

Tokyo - Kuala Lumpur ·

Singapore $\cdot$ Sydney
Beilage zu

Band 40, Heft 1-2, Februar 2017 
Die während des Workshops entwickelten Empfehlungen sind hier zusammengefasst und sollen Kolleginnen und Kollegen bei der Durchführung der Idelalisib-Therapie unterstützen. Im Einzelnen wurden Empfehlungen zu folgenden Bereichen erarbeitet:

- Überwachung von Blutbild und Neutrophilenzahl,

- PJP-Prophylaxe,

- Monitoring auf CMV-Infektion oder Virämie,

- Pneumonitis: Maßnahmen vor Therapiebeginn und Monitoring,

- Maßnahmen bei Diarrhoe und Kolitis.

\section{Vermeidung von Neutropenien}

Hinsichtlich der Neutropenie-Prophylaxe sowie dem Monitoring der Neutrophilenzahl sprach sich das Expertengremium für eine Blutbildbestimmung bei allen Patienten in einem 2-wöchigen Intervall in den ersten 6 Monaten der Idelalisib-Therapie aus. Die absolute Neutrophilenzahl (ANZ) sollte bei Patienten mit einer ANZ $<1000 / \mathrm{mm}^{3}$ mindestens 1-mal pro Woche gemessen werden. Sinkt die ANZ auf $<500 / \mathrm{mm}^{3}$, sollte die Idelalisib-Therapie unterbrochen werden. Steigt die ANZ wieder auf $>500 / \mathrm{mm}^{3}$ an, kann die Behandlung mit einer geringeren Dosierung (100 mg 2-mal/Tag) wieder aufgenommen werden (Tab. 2) [1].

\section{Empfehlung für die PJP-Prophylaxe}

Hinsichtlich der Durchführung einer PJP-Prophylaxe bei Tumorpatienten im Therapieverlauf gibt es in Deutschland bislang keinen Konsens. Zentren, die in der Lymphom- und CLL-Therapie überwiegend Bendamustin verwenden, verzichten auf eine solche Prophylaxe, während diese bei bevorzugtem Einsatz von Fludarabin meist Standard ist. Evidenzbasierte Protokolle der PJP-Prophylaxe existieren bislang nicht. In den mit Idelalisib erstellten Studien wurde eine PJP-Prophylaxe empfohlen, war jedoch nicht vorgeschrieben. Je nach Studie erhielten 20-70\% der Teilnehmer eine derartige Prophylaxe. Der Anteil in CLL-Studien lag mit 40-70\% insgesamt höher [5]. Die Experten sprechen sich bei mit Idelalisib behandelten Patienten für die Gabe von Cotrimoxazol forte (960 mg, Fixkombination aus $800 \mathrm{mg}$ Sulfamethoxazol $+160 \mathrm{mg}$ Trimethoprim) in Dosierungen von 2-3 Tabletten 2-mal pro Woche oder 1-2 Tabletten 3-mal pro Woche aus. Da unbekannt ist, ob fragile Patienten mit Komorbiditäten nicht bereits bei relativ hohen CD4+-T-Zellen gefährdet sind, an einer PJP-Infektion zu erkranken, kann derzeit kein CD4+-Schwellenwert für die Indikation zu einer PJP-Prophylaxe definiert werden. Deshalb sollte diese gemäß Fachinformation unabhängig von der Zahl der CD4+-T-Zellen während der gesamten Idelalisib-Therapie und über einen Zeitraum von 2-6 Monaten nach Behandlungsende durchgeführt werden [1]. Bei Unverträglichkeit von Cotrimoxazol stellt die Inhalation von Pentamidin eine Alternative für die PJP-Prophylaxe dar.

\section{CIMV-Monitoring}

Die aktualisierte Fachinformation empfiehlt bei Patienten mit positiver CMV-Serologie zu Beginn der Behandlung mit Idelalisib eine regelmäßige klinische und Laborüberwachung auf eine CMV-Infektion. Diese Empfehlung gilt auch für Patienten, bei denen sich andere Hinweise auf eine CMV-Infektion in der Vorgeschichte finden lassen. Patienten mit einer CMV-Virämie, bei denen keine entsprechenden klinischen Anzeichen einer CMV-Infektion vorliegen, sind sorgfältig zu überwachen. Bei Patienten mit Hinweisen auf eine CMV-Virämie und klinischen Anzeichen einer CMV-Infektion sollte eine Unterbrechung der Einnahme von Idelalisib erwogen werden, bis die Infektion abgeklungen ist. Wenn der Nutzen einer Wiederaufnahme der Behandlung mit Idelalisib höher eingeschätzt wird als
Tab. 1. Teilnehmer des Experten-Meetings

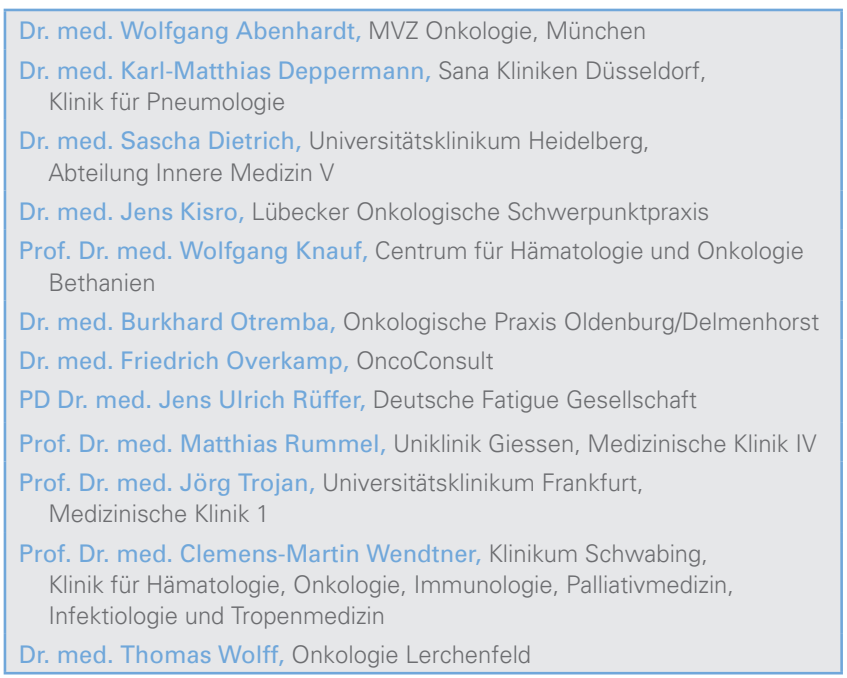

die Risiken, sollte eine vorbeugende CMV-Therapie erwogen werden [1]. Während des Expertenmeetings wurde intensiv über konkrete Empfehlungen in Bezug auf die Methoden zum CMV-Monitoring und dessen Frequenz diskutiert. Demnach sei es derzeit sinnvoll, sich - mangels konkreter Erfahrungen - an den publizierten Empfehlungen des European Conference on Infections in Leukemia (ECIL) zu orientieren $[8,10]$. Generell ist davon auszugehen, dass die Mehrheit der Patienten eine positive CMV-Serologie aufweist. Die Früherkennung einer CMV-Reaktivierung bzw. Virämie wird standardmäßig mittels quantitativer Polymerase-Kettenreaktion (qPCR) durchgeführt. Hierbei sollte ein Ergebnis mit einer Kopienzahl von > 500$1000 \mathrm{Kopien} / \mathrm{ml}$ als positiv für CMV gewertet werden. Alternativ ist auch die Bestimmung des pp65-Antigens möglich. Für die Frequenz des Monitorings gibt es derzeit keine allgemeingültigen Vorgaben. Zur Orientierung erachteten die Experten eine 2-wöchentliche Testung für die ersten 8-12 Wochen der Therapie als empfehlenswert. Bei unauffälligen Befunden und nach Abwägung der patientenindividuellen Risiken sind dann längere, $z . B$. monatliche Intervalle, möglich. Zudem sollten Patienten dahingehend unterrichtet werden, bei Anzeichen für eine Infektion, z.B. Fieber, vorstellig zu werden. Für die gezielte Therapie einer CMV-Infektion ist nach Meinung der Experten eine Therapie mit Valganciclovir in einer Tagesdosis von 900 mg angezeigt.

\section{Vorgehen bei früher und später Diarrhoe sowie bei Kolitiden}

Im Verlauf einer Idelalisib-Therapie können eine schwere Diarrhoe (Grad $\geq 3$ ) und Kolitiden auftreten. In den mit Idelalisib erstellten

Tab. 2. Überwachung der Neutrophilenzahl, modifiziert nach [1]

\begin{tabular}{|lll|}
\begin{tabular}{|ll} 
ANZ 1000 bis \\
$<1500 / \mathrm{mm}^{3}$
\end{tabular} & $\begin{array}{l}\text { ANZ } 500 \text { bis } \\
<1000 / \mathrm{mm}^{3}\end{array}$ & ANZ $<500 / \mathrm{mm}^{3}$ \\
\hline $\begin{array}{ll}\text { Einnahme von } \\
\text { Zydelig fortsetzen }\end{array}$ & $\begin{array}{l}\text { Einnahme von } \\
\text { Zydelig fortsetzen }\end{array}$ & $\begin{array}{l}\text { Einnahme von Zydelig unter- } \\
\text { brechen }\end{array}$ \\
& ANZ mindestens & ANZ mindestens 1-mal wöchent- \\
& 1-mal wöchentlich & lich überprüfen, bis die ANZ einen \\
& überprüfen & Wert von $\geq 500 / \mathrm{mm}^{3}$ erreicht. \\
& Danach kann die Einnahme von \\
& Zydelig mit einer Dosierung von \\
& 100 mg 2-mal täglich wieder- \\
& aufgenommen werden. \\
\hline
\end{tabular}


Studien lagen die Raten an Diarrhoe vom Grad $\geq 3$ zwischen 5 und $17 \%$ [6]. Generell werden Diarrhoen und Kolitiden median 1,9 Monate nach Therapiebeginn dokumentiert. Während sich eine leichtere Diarrhoe (Grad 1-2) eher früh - nach median 1,5 Monaten - entwickelt, wird eine Diarrhoe vom Grad $\geq 3$ verzögert nach median 7,1 Monaten beobachtet [6]. Für das Management von Diarrhoen und Kolitiden wurden im letzten Jahr Empfehlungen von Coutré et al. [6] erarbeitet. Die Empfehlungen dieser Expertengruppe (Tab. 1) für die früh auftretenden leichten bzw. die verzögert einsetzenden schweren Diarrhoen lehnen sich an die der US-amerikanischen Arbeitsgruppe an [6]. Die Experten differenzieren zwischen leichten bis mäßig schweren Diarrhoen vom Grad 1-2, die früh in den ersten 8 Therapiewochen auftreten, meist selbstlimitierend sind und auf Medikamente wie Loperamid ansprechen (Abb. 1) [7]. Bei den früh auftretenden leichteren Diarrhoen (Grad 1-2) reichen üblicherweise diätetische Maßnahmen wie ausreichende Flüssigkeitszufuhr und häufige kleine Mahlzeiten (z.B. Banane, Reis, Toast) und die Gabe von Loperamid (initial $4 \mathrm{mg}$, dann $2 \mathrm{mg}$ alle 4 Stunden bzw. nach jedem flüssigen Stuhl). Haben sich die Durchfälle nach 24-48 Stunden rückgebildet, kann schrittweise wieder normale Nahrung aufgenommen und Loperamid nach einem 24-stündigen Diarrhoefreien Intervall abgesetzt werden (Abb. 1) [7]. Problematischer ist der zweite, meist erst später im Therapieverlauf auftretende Diarrhoe-Typ, der kaum auf Peristaltikhemmer anspricht. Diese Durchfälle sind meist wässrig, frei von Blut- und Schleimbeimengungen und gehen nicht mit Krämpfen einher. Koloskopisch fallen teilweise histologische Veränderungen gemäß einer lymphozytären Kolitis auf. Der ursächliche Mechanismus für diese späte und schwere Diarrhoe ist bisher unklar. Als diagnostische Maßnahmen bei Auftreten einer Diarrhoe werden Stuhluntersuchungen zum Ausschluss einer infektiösen Ursache und die Koloskopie empfohlen. Bei anhaltender Diarrhoe gelten ebenso wie bei der schweren Diarrhoe vom Grad 3-4 spezifische Maßnahmen (Abb. 2). Bei Hinweisen für eine Exsikkose sollte frühzeitig eine intravenöse (i.v.) Flüssigkeitssubstitution erfolgen. Nach Ausschluss infektiöser Ursachen und Absetzen von Idelalisib sollte eine Therapie mit Budesonid oder i.v. verabreichtem hoch dosiertem Steroid (2-3 $\mathrm{mg} / \mathrm{kg}$ Körpergewicht (KG)) initiiert werden. Die Steroidgabe ist bis zum kompletten Sistieren der Durchfälle bzw. über maximal 4 Tage fortzuführen. Danach kann ein Ausschleichen von Budesonid bzw. des i.v. verabreichten Steroids erwogen werden. Allerdings wurden mittlerweile auch fulminante Diarrhoen beobachtet, die trotz i.v. verabreichter Steroide therapierefraktär sind. In diesem Fall sprachen sich die deutschen Experten für die Gabe eines Tumornekrosefaktor alpha (TNF- $\alpha$ )-Inhibitors (Infliximab, Adalimumab) in Analogie zur Behandlung schwerer, steroidrefraktärer immunvermittelter Kolitiden unter Immuncheckpoint-Inhibitortherapie aus [9], der allerdings in dieser Indikation nicht zugelassen ist.

\section{Pneumonitis}

In klinischen Studien wurden bei 3\% der mit Idelalisib behandelten Patienten nichtinfektiöse Pneumonitiden beobachtet [7]. Die deutsche Expertenrunde spricht sich dafür aus, vor Beginn der Idelalisib-Therapie bei allen Patienten auch ohne bekannte Lungenerkrankungen eine Lungenfunktionsprüfung mit Ermittlung der Diffusionskapazität durchzuführen. Diese Untersuchung ist auch für die Verlaufsbeobachtung wichtig, da bei Auftreten von Atemwegsbeschwerden ein Ausgangsbefund vorliegt. Bei normaler Lungenfunktion kann die Therapie mit Idelalisib begonnen werden. Bei restriktiver Ventilationsstörung, Vorliegen von Atemwegssymptomen und bei begleitender KHK sollte eine intensivere Diagnostik inklusive Thorax-High-Resolution-Computertomographie (Thorax-HR-CT) angestrebt werden, um die zugrunde liegende Ursache abzuklären. Entwickeln sich Atemwegsbeschwerden wie Dyspnoe und/oder Husten im Verlauf der Idelalisib-Therapie, wird eine weitere Diagnostik inklusive einer erneuten Lungenfunktionsprüfung empfohlen. Ergeben Röntgen-Thorax und CT im Anschluss einen auffälligen Befund, ist eine Bronchoskopie mit bronchoalveolärer Lavage indiziert. Bei Ausschluss opportunistischer Infektionen und atypischer Pneumonien ist eine Pneumonitis wahrscheinlich. In diesem Fall muss Idelalisib abgesetzt und eine Therapie mit Steroiden initiiert werden [1]. Die genaue Steroiddosierung ist derzeit unklar. Bei einer Pneumonitis unter Immuncheckpoint-Blockade werden je nach Schweregrad Dosierungen von 0,5 bis $2 \mathrm{mg} / \mathrm{kg}$ KG empfohlen [9]. Nach dem Abklingen der Pneumonitis kann eine Wiederaufnahme der Behandlung mit Idelalisib mit einer Dosierung von $100 \mathrm{mg}$ 2-mal täglich in Betracht gezogen werden [1].

Management (Diarrhoen Grad 1-2)

- Anamnese und physische Untersuchung der Patienten, um eine Infektion auszuschließen

- Patienten-Empfehlung:

- Keine Laktose haltigen Lebensmittel, kein Alkohol, keine hoch-osmolaren Nahrungsmittelergänzungsmittel - eine gute Flüssigkeitszufuhr sicherstellen (8-10 große Gläser am Tag)

- regelmäßig kleine Malzeiten einnehmen (z.B. Bananen, Reis, Apfelmus, Toast, Nudeln ohne Soße)

- Anzahl der Stuhlgänge erfassen, dem Arzt Symptome lebensbedrohlicher Spätfolgen, z.B. Fieber und/oder Schwindel melden

$\downarrow$

Behandlung

- Loperamid, initiale Dosierung 4 mg, nachfolgend alle 4 Stunden 2 mg oder nach jedem weichen Stuh

$\downarrow$

Folgewertung nach 24-48 Stunden

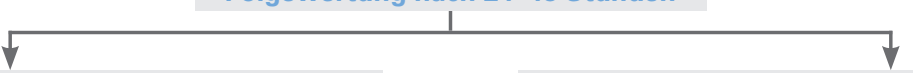

Diarrhoe erfolgreich behandelt

Diarrhoe nicht erfolgreich behandelt

- Diätetische Maßnahmen beibehalten

- Zur Diät schrittweise feste Nahrung hinzufügen

- Loperamid nach erinem Diarrhoe-freien Intervall von

12 Stunden absetzen

Abb. 1. Algorithmus für das Management von Diarrhoen Grad 1-2, modifiziert nach [7]. 
- Untersuchung des Patienten, um eine Infektion als Ursache auszuschließen

- Die Behandlung mit Idelalisib aussetzen (siehe Fachinformation)

- Diätetische Maßnahmen sicherstellen

- Intravenöse Flüssigkeitssubstitution oder orale Flüssigkeitszufuhr bei Zeichen einer Dehydratation oder Grad $\geq 3$ Diarrhoe oder Kolitis

$\downarrow$

Behandlung, wenn infektiöse Ätiologie ausgeschlossen

- Budesonid: 3 Hartkapseln à 3 mg 1-mal täglich oder

- orale Steroide: Prednison (wenn Diarrhoe Grad 1 erreicht, allmählich ausschleichen) oder

- i.v. Steroide, wenn der Patient eine i.v. Flüssigkeitstherapie erhält oder keine orale Medikation toleriert

\section{$\downarrow$}

Patient toleriert eine orale Medikation

- Umstellung von i.v. Steroidtherapie auf Budesonid oder orale Steroide

$\downarrow$

Diarrhoe Grad $\leq 1$ erreicht

- Diätetische Maßnahmen beibehalten

- Zur Diät schrittweise feste Nahrung hinzufügen

- Ausschleichen von Budesonid und oralen Steroiden in Betracht ziehen

- Die Behandlung mit Idelalisib wiederaufnehmen bei einer Dosierung mit 100 mg 2-mal täglich (siehe Fachinformation) und

- Begleitende Therapie mit Budesonid in Betracht ziehen (siehe Fachinformation)

Abb 2. Algorithmus für das Management später komplizierter Diarrhoen Grad 3-4, modifiziert nach [7].

\section{Fazit}

Die Entwicklung zielgerichtet wirkender Medikamente ist als großer Fortschritt in der Therapie solider und hämatologischer Tumoren zu werten. Die Effektivität der Behandlung wurde deutlich verbessert. Beim Schritt von der klinischen Forschung in die tägliche Praxis traten allerdings auch neue, aus der ZytostatikaTherapie nicht bekannte Nebenwirkungen auf. Diese Erkenntnis bietet die Chance, den Einsatz von Idelalisib zu optimieren und weitere Patientengruppen zu identifizieren, die von dieser Substanz besonders profitieren können. Es ist wichtig, umfassende klinische Erfahrungen zum Management dieser unerwünschten Begleiterscheinungen zu sammeln und Algorithmen zu entwickeln, um Idelalisib optimal einsetzen zu können. Es ist zu hoffen, dass die auf dem Expertenmeeting erarbeiteten Empfehlungen zum Management der Idelalisib-Therapie weite Verbreitung finden und für Hämatologen und Onkologen beim Einsatz dieser neuen Substanz im klinischen Alltag hilfreich sind.

Dr. Katharina Arnheim, Freiburg i.Br.

\section{Zitate}

Prof. Dr. Rummel: "Es wäre wünschenswert, den Pathomechanismus bei der CMV-Reaktivierung und PJP-Pneumonie aufzuklären, um danach gezielte Prophylaxemaßnahmen entwickeln zu können.»

Prof. Dr. Wendtner: "Die CMV-Reaktivierung ist kein substanzspezifisches Problem, sondern wurde auch in derzeit laufenden Studien bei Kombination eines Bcl-2-Inhibitors mit einem Anti-CD20-Antikörper in der Folge einer schweren Neutropenie beobachtet."

Prof. Dr. Jörg Trojan: «Bei Patienten mit schwerer Diarrhoe sollte eine Koloskopie, zumindest aber eine Sigmoidoskopie durchgeführt werden, um eine eventuelle CMV-Kolitis auszuschließen. Wissenschaftlich ist zudem von Interesse, ob Kolitiden unter Idelalisib histologisch tatsächlich durch eine lymphozytäre Infiltration charakterisiert sind.»

\section{Quelle}

Expertenmeeting «Therapiemanagement mit Zydelig», Frankfurt, 20. April 2016; Veranstalter: Gilead Sciences GmbH.

\section{Literatur}

1 Zydelig ${ }^{\circledR}$ Fachinformation, Stand November 2016.

2 Furman RR et al.: N Engl J Med 2014;370:997-1007.

3 Sharman JP et al.: ASH 2014; abstr 330.

4 Salles G et al.: ASCO 2015; abstr 8529.

5 European Medicines Agency: EMA Assessment Report; procedure under Article 20 of regulation (EC) No 726/2004 resulting from pharmacovigilance data, 7. Juli 2016. www.ema.europa.eu/docs/en_GB/document_library/Referrals_document/ Zydelig_20/European_Commission_final_decision/WC500213555.pdf.

6 Zydelig ${ }^{\circledR}$ Rote-Hand-Brief: Wichtige Information zu Zydelig, Einschränkungen für die Anwendung von Zydelig (Idelalisib) zur Behandlung der chronischen lymphatischen Leukämie (CLL) und des rezidivierten follikulären Lymphoms (FL) aufgrund neuer Erkenntnisse aus klinischen Studien. Gilead Sciences GmbH, Martinsried/München, 22. August 2016.

7 Coutré SE et al.: Leuk Lymphoma 2015;56:2779-2786.

8 Ljungmann P et al.: Bone Marrow Transplant 2008;42:227-240.

9 Spain L et al.: Cancer Treat Rev 2016;44:51-60.

10 Ljungman $\mathrm{P}$ et al.: Recommendations for CMV and HHV-6 management in patients with hematological diseases ECIL. 4th European Conference on Infections in Leukemia (ECIL-4); accessed September 26, 2011.

\section{Impressum}

Konsensusempfehlungen deutscher Experten

\section{Management der Therapie mit Idelalisib}

Beilage zu Oncology ReSEARCH AND TREATMENT 40 | 1-2 | 17

Mit freundlicher Unterstützung durch

\section{GILEAD Sciences GmbH}

Die Wiedergabe von Gebrauchsnamen, Handelsnamen, Warenbezeichnungen usw. in dieser Zeitschrift berechtigt auch ohne besondere Kennzeichnung nicht zur Annahme, dass solche Namen im Sinne der Warenzeichen- und Markenschutz-Gesetzgebung als frei zu betrachten wären und daher von jedermann benutzt werden düren. Für Angaben von Dosierungsan be von jedenan bentzt werden dur. formen kann vom Verlag keine Haftung übernommen werden. Derartige Angaben müssen vom jeweligen Anwender im Einzelfall anhand anderer Literaturstellen auf ihre Richtigkeit überprüft werden.
Ohne schriftliche Genehmigung des Verlags dürfen diese Publikation oder Teile daraus nicht in andere Sprachen übersetzt oder in irgendeiner Form mit mechanischen oder elektronischen Mitteln (einschließlich Fotokopie, Tonaufnahme oder Mikrokopie) reproduziert oder auf einem Datenträger oder Computersystem gespeichert werden.

Alle Rechte vorbehalten.

() 2017 by S. Karger Verlag für Medizin und Naturwissenschaften GmbH, Postfach, 79095 Freiburg, Deutschland.

Druck: Kraft Premium GmbH, 76275 Ettlingen, Deutschland. 